\title{
Performance of the ATLAS Tile Calorimeter
}

\author{
Mpho Gift Doctor Gololo, on behalf of the ATLAS Collaboration \\ University of the Witwatersrand, \\ Johannesburg, South Africa \\ E-mail: mpho.gift.doctor.gololo@cern.ch
}

\begin{abstract}
The TileCal is a sampling hadronic calorimeter covering the central region of the ATLAS experiment, with steel as absorber and plastic scintillators as active medium. The scintillators are read out by wavelength shifting fibres to PMTs at the back of each wedge-shaped calorimeter module. The analogue signals from the PMTs are amplified, shaped, and digitised on the detector every $25 \mathrm{~ns}$, and stored on detector in digital pipeline buffers until a trigger decision is received. The data are then read out to the off-detector systems for further processing. The TileCal employs several calibration systems that, together with the collected collision data, provide the basis for response equalisation and monitoring at each stage of the readout path; from scintillation light production to energy and time reconstruction. Furthermore, the calorimeter performance has been established with large samples of proton-proton collision data during LHC Run 1 and Run 2. The highmomentum isolated muons have been used to study and validate the electromagnetic scale, while the hadronic response has been probed with isolated hadrons. The calorimeter time resolution has been studied with multi-jet events. We present and summarise results of the calorimeter calibration and performance.
\end{abstract}

Keywords: Calorimeters; Performance of High Energy Physics detectors; Calibration systems 


\section{Contents}

1 Introduction 1

2 Calibration Systems $\quad 1$

2.1 Charge Injection System (CIS) 3

2.2 Cesium Calibration System 3

2.3 Laser System 4

2.4 Minimum Bias System 5

3 Performance 5

3.1 Detector Status and Data Quality 5

3.2 Noise, Single Particle and Muon Response 6

$\begin{array}{llr}4 & \text { Conclusion } & 8\end{array}$

\section{Introduction}

The Large Hadron Collider (LHC) [1] collides proton beams at four interaction points where the main experiments are located. These are: ATLAS (A Toroidal LHC ApparatuS) [2], CMS (Compact Muon Solenoid), ALICE (A Large Ion Collider Experiment) and LHCb (Large Hadron Collider beauty). The Tile Calorimeter (TileCal) [3] is the central hadronic calorimeter of the ATLAS experiment at the LHC. It is partitioned into three physical volumes: a long barrel (LB) that spans a pseduorapidity region of $0<|\eta|<1.0$ and two extended barrels (EB) each spanning a region of $0.8<|\eta|<1.7$ as shown in Figure 1(a).

The TileCal is used to provide ATLAS Level-1 trigger information and also contributes to the measurement of the energy and position of the jets and the missing transverse energy. It is a sampling calorimeter that uses steel absorber and scintillating plastic tiles as active material as shown in Figure 1(b). The light is produced in scintillating tiles, collected and routed by wavelength shifting fibers and converted into electric signals by the PMTs with two different gains by a factor 64.

\section{Calibration Systems}

Different dedicated calibration systems are used to monitor the behavior of the different detector components. Equation 2.1 shows the reconstructed energy of each TileCal channel, $E[\mathrm{GeV}]$, derived from the raw response, $A[\mathrm{ADC}]$, as follows:

$$
E[\mathrm{GeV}]=A[\mathrm{ADC}] \cdot C_{\mathrm{ADC} \rightarrow \mathrm{pC}} \cdot C_{\mathrm{pC} \rightarrow \mathrm{GeV}} \cdot C_{\mathrm{Cs}} \cdot C_{\mathrm{Las}}
$$




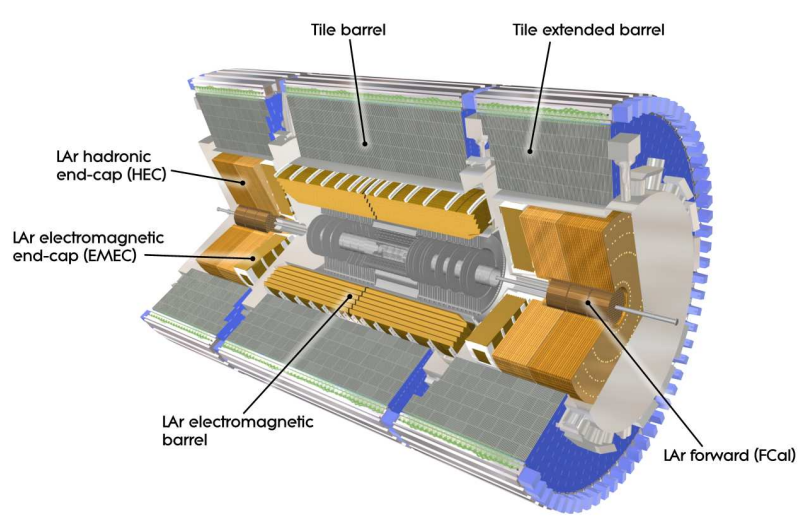

(a)

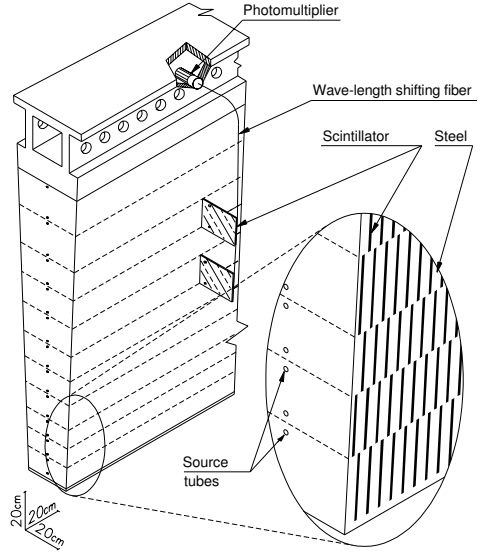

(b)

Figure 1: (a) A cut-away diagram of the ATLAS inner detector and calorimeter. The TileCal consists of one barrel and two extended barrel sections. (b) A total of 256 such modules make up the full TileCal [4].

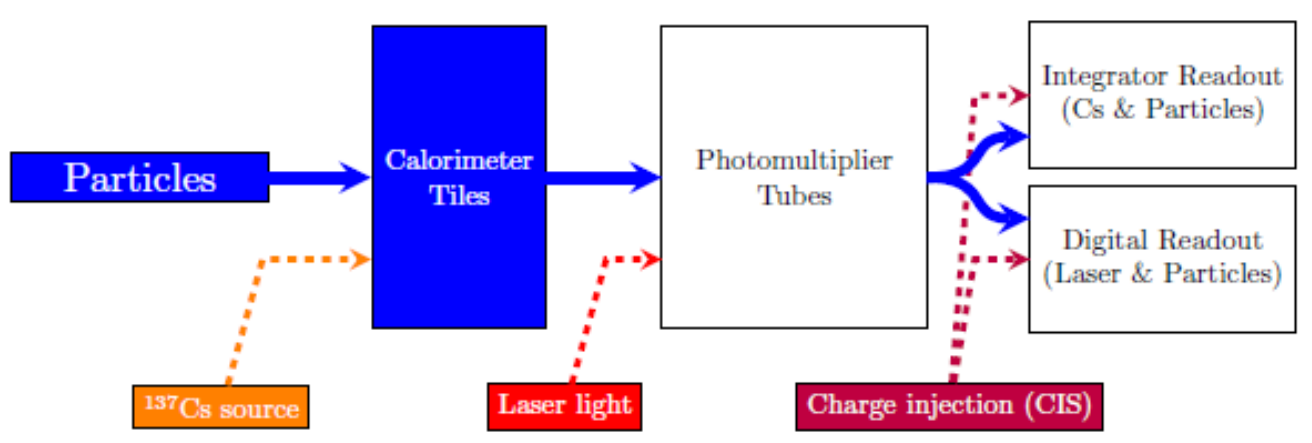

Figure 2: Flow diagram of the readout signal path of the different TileCal calibration tools. The physics signal is denoted by the thick solid line and the path taken by each of the calibration systems is shown with dashed lines [5],[6].

All the different $C$ factors shown in the equation represents the calibration constants. The variations in PMT high-voltage, stress induced on the PMTs by high light flux or optics aging impact the factors to evolve with time. The calibration systems are used to monitor the stability of these factors and provide corrections for each channel. The calibration constants are provided by individual systems during the ATLAS operations:

- Charge Injection System (CIS): Calibrates the response of ADCs (electronics): $C_{\mathrm{ADC} \rightarrow \mathrm{pC}}$

- The electromagnetic scale calibration constant: converts the calorimeter signals measured as electric charge in $\mathrm{pC}$ to the energy deposited by measured electrons which produced the signals, was fixed during dedicated test beam campaigns [7]: $C_{\mathrm{pC} \rightarrow \mathrm{GeV}}$

- Cesium system: a moveable Cs-137 source to calibrate the optic components and the PMTs: $C_{\mathrm{Cs}}$ 


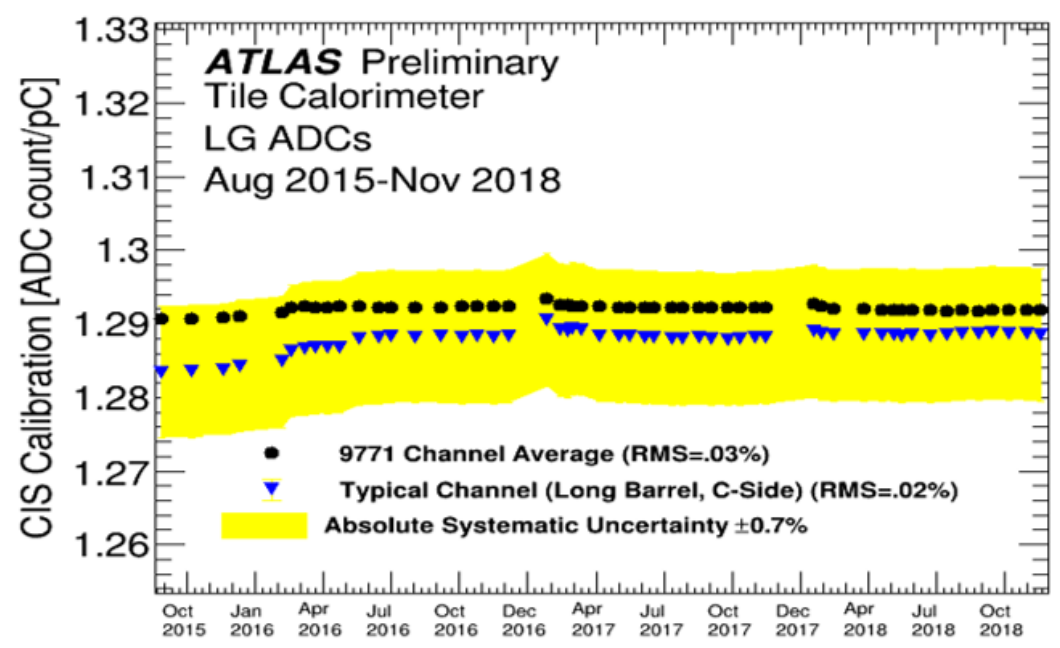

Figure 3: The CIS constants from a typical channel. The RMS value in the Figure $(0.03 \%)$ corresponds to the channel fluctuations present in calibrations. In addition, there is a $0.7 \%$ systematic uncertainty present in individual calibrations, represented by the yellow band [6].

- Laser System: Calibrates variations due to electronics and PMTs: $C_{\text {Las }}$

- Minimum Bias System (MB): Monitors beam conditions, optical components and PMT gains.

\subsection{Charge Injection System (CIS)}

This calibration system is used to calibrate the response of ADCs (electronics) by injecting a signal of known charge. A conversion factor is then extracted from $\mathrm{ADC}$ counts to $\mathrm{pC}, C_{\mathrm{ADC} \rightarrow \mathrm{p} C}$, by spanning the full $\mathrm{ADC}$ range of $0-800 \mathrm{pC}$ and monitoring both the low and high gain of all channels. The overall stability of the calibration factor is at the level of $0.03 \%$ with a precision of $0.7 \%$ for the full LHC Run-2 as shown in Figure 3. The corresponding calibration runs are taken from daily to weekly, and the linearity is well contained within the uncertainty

\subsection{Cesium Calibration System}

The Cesium system is based on three moveable radioactive sources using a hydraulic control through a system of steel tubes. The Cs-137 sources move inside the calorimeter, emitting 0.662 $\mathrm{MeV}$ photons to illuminate the scintillators. The channel response to the energy deposits is used to equalize the response of all the cells and maintain global response of the calorimeter at the electromagnetic scale. Between Run 1 and Run 2, a new water storage system, lower pressure in the hydraulics, and more precise water level metering during the scans were installed to improve stability and safety of the operation. Cell response is not constant in time due to the PMT gain variation and scintillator degradation due to the exposure to beam as shown in Figure 4. The precision of the system in a single typical cell is approximately $0.3 \%$, and the variation of the $C_{\mathrm{Cs}}$ during Run 2 is $10 \%$ for the first radial layer, closest to the beam pipe, and less than $4 \%$ for the other two layers. 


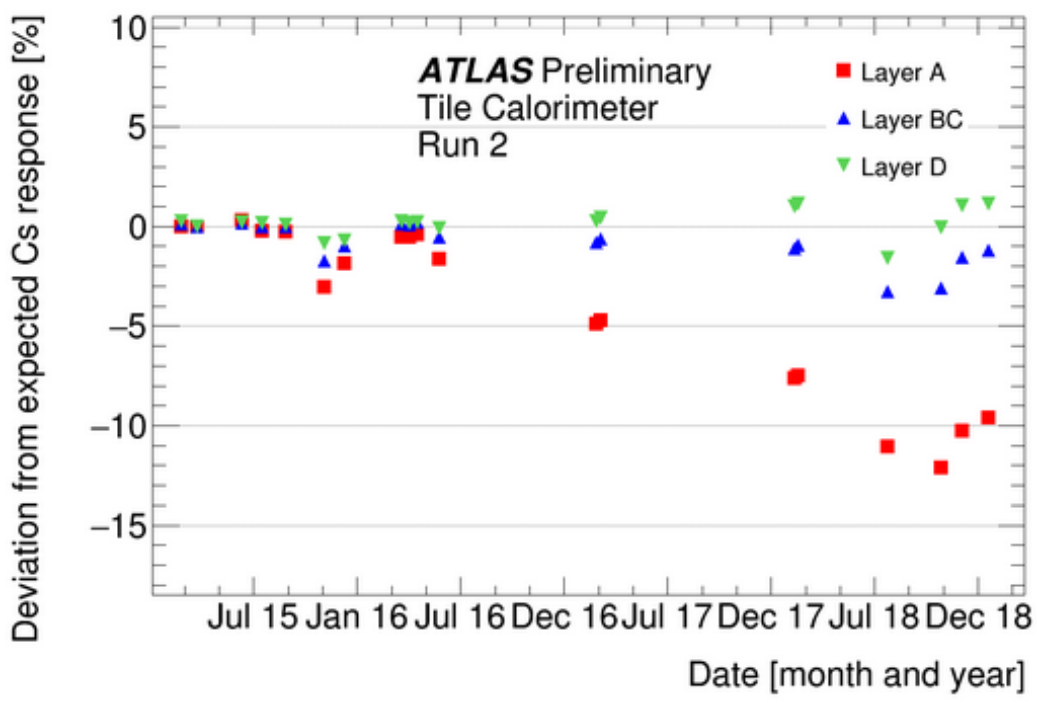

Figure 4: The variation in TileCal response measured by the Cesium calibration system. The points represent the average values of the responses of the cells in a given layer [6].

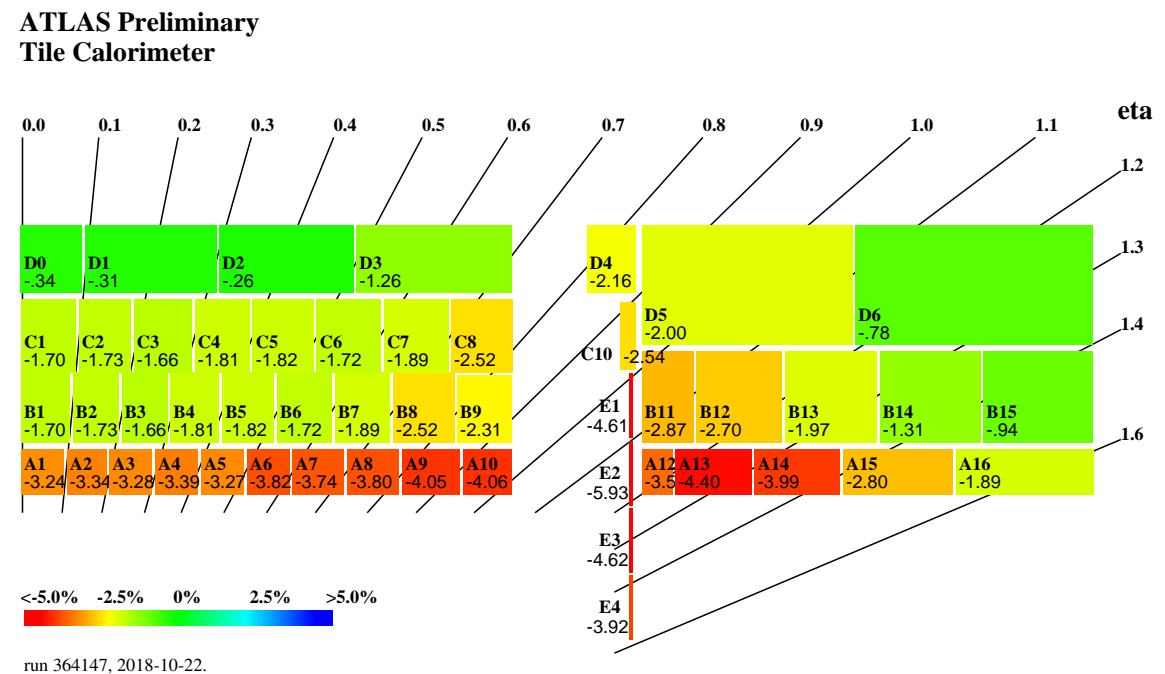

Figure 5: The drift after the full proton-proton data taking period in 2018 showing the mean gain variation (\%) in the TileCal cells, as a function of $\eta$ and radius. The observed down-drift mostly affects cells at inner radii, which are the cells with higher current [8].

\subsection{Laser System}

The Laser system measures the gain stability of the PMTs by sending a controlled amount of light into each PMT at a wavelenth of $532 \mathrm{~nm}$. The gain variation is measured between two Cesium scans where by the Laser measures the drift seen in the PMTs with respect to the last Cesium scan. 

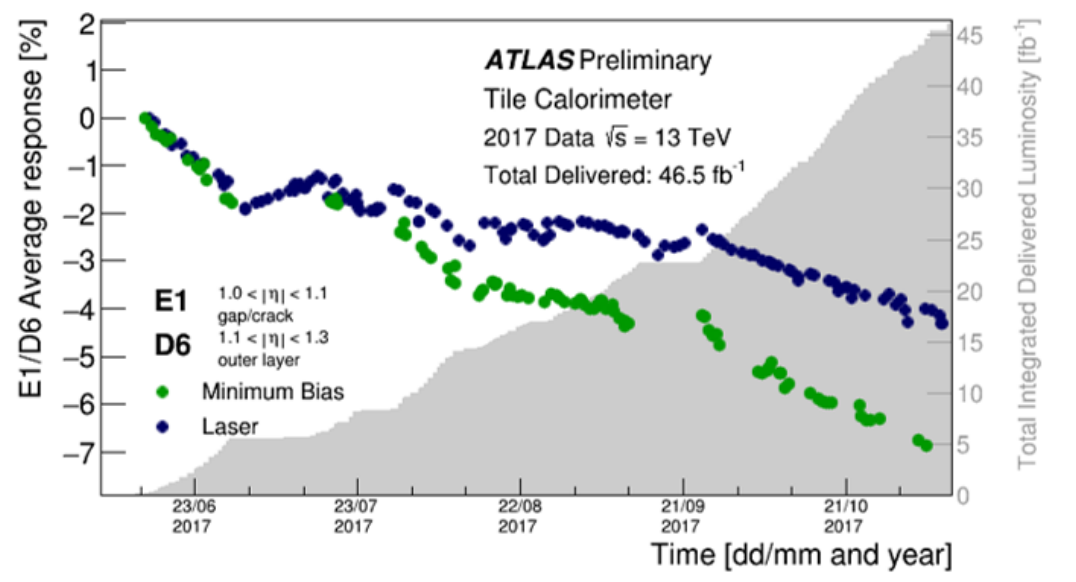

Figure 6: Variations in the response to Minimum Bias and Laser for cells in the inner layer of the extended barrel as functions of time during the 2017 data taking period [9].

Deviations in a channel's response with respect to its nominal value (at the time of the latest Cesium calibration) is then translated into a calibration constant: $C_{\mathrm{Las}}$. A new Laser system was developed to correct shortcomings in electronics and light monitoring of the first system. This resulted in an improved resolution that shows a typical resolution on the gain variation to be better than $0.5 \%$ per channel. Figure 5 shows the mean gain variation per cell type observed during proton-proton data taking in 2018. The variation of this constant went up to 6\% in 2018.

\subsection{Minimum Bias System}

The so-called Minimum Bias events, high energy proton-proton collisions dominated by soft parton interactions, are used by this calibration system to monitor the full optical chain. The system is used to measure instantaneous luminosity given an initial calibration. The difference between Minimum Bias and Laser is interpreted as an effect of the scintillators' irradiation. Figure 6 shows down-drifts observed during collisions and up-drifts during maintenance periods and machine developments, for the least (D6) and most exposed (E1) cell types. This Figure shows the variations in the response of the most highly irradiated regular cells observed by Minimum Bias monitoring and Laser systems during the 2017 data taking period.

\section{Performance}

\subsection{Detector Status and Data Quality}

Monitoring of the TileCal is performed in order to identify and mask problematic channels by correcting monitoring data corruption, timing jumps or other hardware issues. These issues are fixed during maintenance periods to allow good recovery of the system. The fraction of the masked cells at the end of each year is decreasing as shown in Figure 7 (a). The shaded regions correspond to maintenance periods, when the front-end electronics could be accessed and repaired. The red line in figure 7 (b) corresponds to a complete module that was not operational due to a cooling 


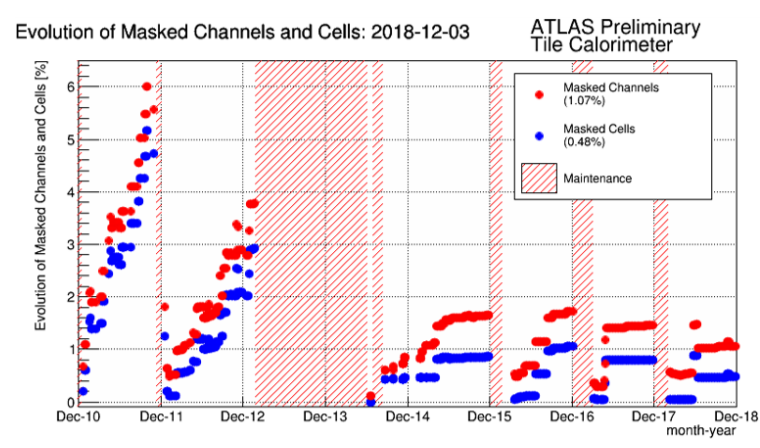

(a)

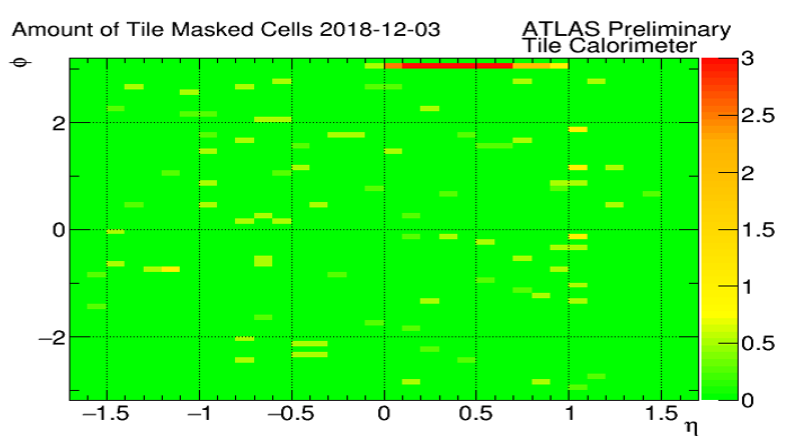

(b)

Figure 7: (a) The percentage of masked cells and channels as functions of time from December 2010 to December 2018. (b) The amount of Tile masked cells for the year 2018 [10].

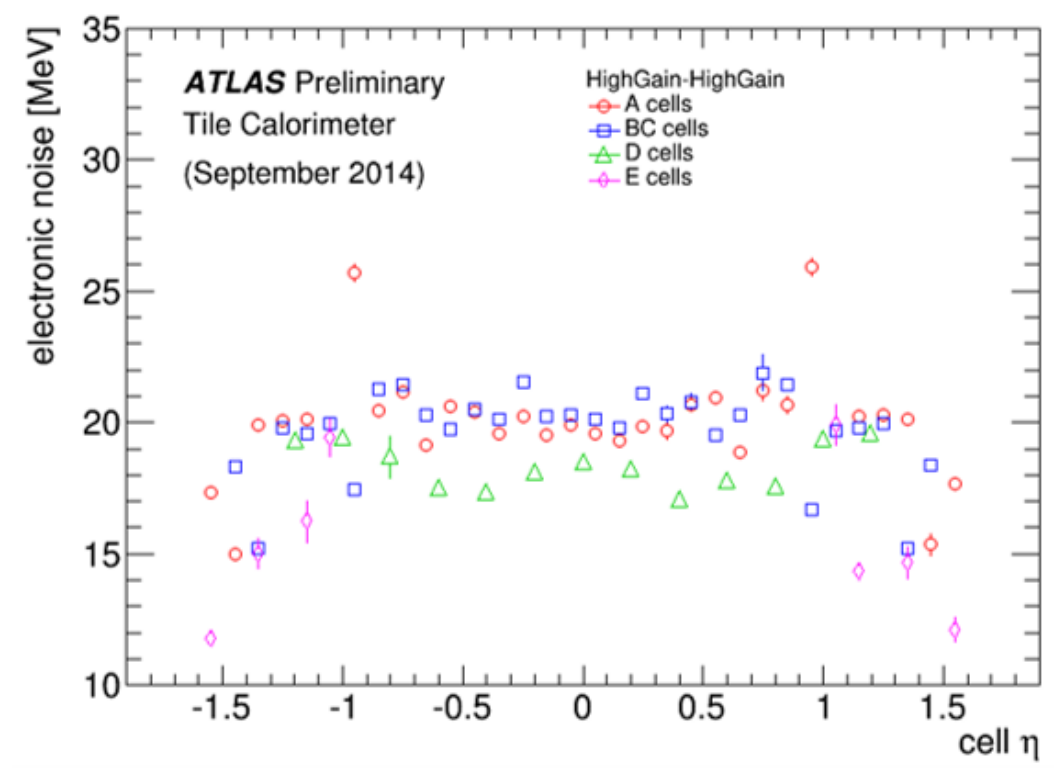

Figure 8: Electronic noise measured in the TileCal cells as a function of $\eta$. [11].

malfunction. The TileCal achieved $100 \%$ data quality efficiency in $2015,99.3 \%$ in $2016,99.4 \%$ in 2017 and $100 \%$ in 2018.

\subsection{Noise, Single Particle and Muon Response}

The total noise per cell in the calorimeter comes from two sources: electronic noise and pile-up contribution. The electronic noise is measured in dedicated runs with no signal in the detector and the pile-up contribution originates from multiple interactions occurring at the same bunch crossing or from the minimum bias events from previous/following bunch crossings. It contributes to the signal response and to the widening of the cell energy distribution, increasing with the average number of interactions per bunch crossing, $\langle\mu\rangle$. Figure 8 shows that the electronics noise stays at a level below $20 \mathrm{MeV}$ for most of the cells and Figure 9 shows the increase of the total noise, 


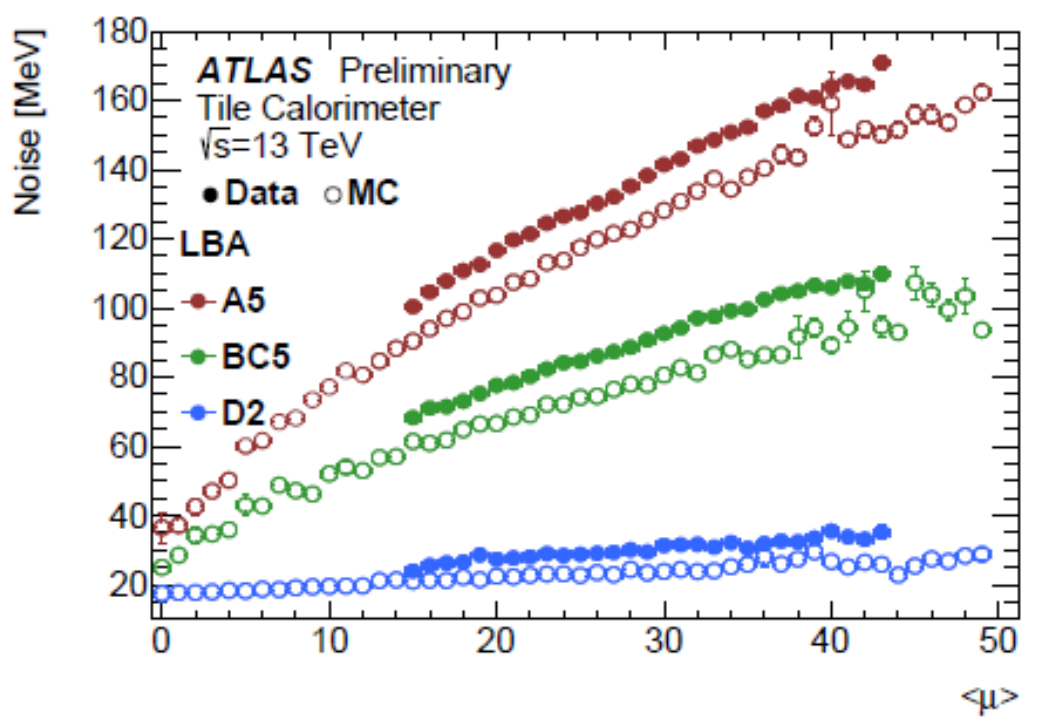

Figure 9: Total noise measured in the TileCal cells in data and MC as functions of $\langle\mu\rangle$ [12].

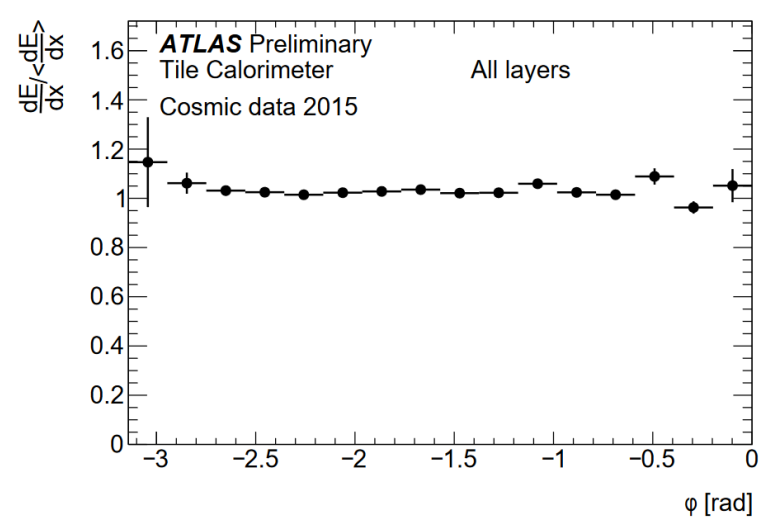

(a)

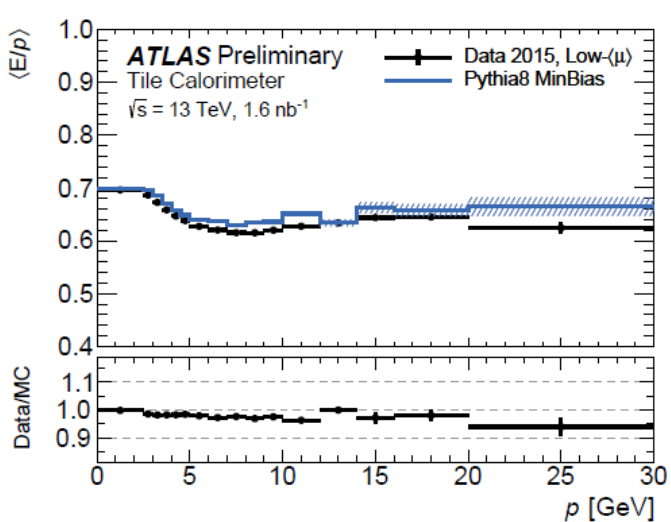

(b)

Figure 10: (a)Uniformity of the muon energy loss per unit distance, $\mathrm{dE} / \mathrm{dx}$, as a function of $\phi$ using 2015 cosmic data (b) Calorimeter response to single isolated charged hadrons as a function of momentum measured in $\sqrt{s}=13 \mathrm{TeV}$ data and Monte Carlo [13], [14].

defined as the standard deviation of the cell energy distribution, as a function of $\langle\mu\rangle$. Figure 10 (a) shows the energy deposited by cosmic muons normalized to the average response. Deviations from unity correspond to angles with smaller statistics. Figure 10 (b) shows the mean $E / p$ as a function of track momentum measured in data and in MC simulation. The simulation agrees with the data within 3\%, showing uniformity in detector response. Cell response is estimated as the energy deposited by the muon per length of the track path $(\mathrm{d} E / \mathrm{d} x)$. 


\section{Conclusion}

The TileCal is an important part of the ATLAS detector at the LHC, and provides information for the reconstruction of hadrons, jets, hadronic decays of tau leptons and missing transverse energy. Multiple systems are used to calibrate and monitor the response of the TileCal cells. Inter-calibration and uniformity are monitored with isolated charged hadrons and cosmic muons. These calibration systems allowed a great performance of the calorimeter to be achieved. The stability of the absolute energy scale at the cell level was maintained to be better than $1 \%$ during Run 2 data taking.

\section{References}

[1] L. Evans and P. Bryant. The LHC: Accelerator and Experiments. 2008 JINST 3 S08001. https://iopscience.iop.org/article/10.1088/1748-0221/3/08/S08001/meta.

[2] The ATLAS Collaboration. The ATLAS Experiment at the CERN Large Hadron Collider. JINST, 3:S08003, 2008. https://iopscience.iop.org/article/10.1088/1748-0221/3/08/S08003/meta.

[3] The ATLAS Collaboration, ATLAS Tile Calorimeter Technical Design Report, CERN-LHCC-96-042. http://cds.cern.ch/record/331062.

[4] The ATLAS Collaboration, Approved Tile Calorimeter Plots (2020) https://twiki.cern.ch/twiki/bin/view/AtlasPublic/PublishedTilecalFigures.

[5] The ATLAS Collaboration. Operation and performance of the ATLAS Tile Calorimeter in Run 1, Eur. Phys. J. C78 (2018) 987, 2018, http://cds.cern.ch/record/2622093.

[6] The ATLAS Collaboration, Approved Tile Calorimeter Calibration Group Plots (2020) https://twiki.cern.ch/twiki/bin/view/AtlasPublic/ApprovedPlotsTileCalibration.

[7] P. Adragna et al., Testbeam studies of production modules of the ATLAS Tile Calorimeter. Nucl. Instrum. Meth. A 606 362, 2009.

https://www.sciencedirect.com/science/article/pii/S016890020900792X.

[8] The ATLAS Collaboration, Approved Tile Calorimeter Calibration Laser Plots (2020) https://twiki.cern.ch/twiki/bin/view/AtlasPublic/ApprovedPlotsTileCalibrationLaser.

[9] The ATLAS Collaboration, Public Tile Calorimeter Combined Calibration Plots for Collision Data (2020) https://twiki.cern.ch/twiki/bin/view/AtlasPublic/ApprovedPlotsTileCalibration.

[10] The ATLAS Collaboration, Public Tile Calorimeter Detector Status Plots (2020) https://twiki.cern.ch/twiki/bin/view/AtlasPublic/ApprovedPlotsTileDetectorStatus.

[11] The ATLAS Collaboration, Public Tile Calorimeter Electronic Noise Plots (2020) https://twiki.cern.ch/twiki/bin/view/AtlasPublic/ApprovedPlotsTileNoise.

[12] The ATLAS Collaboration, Public Tile Calorimeter Pile-Up Noise Performance Plots Tile (2020) https://twiki.cern.ch/twiki/bin/view/AtlasPublic/ApprovedPlotsTilePileUpNoisePerformance.

[13] The ATLAS Collaboration, Public Tile Calorimeter Energy Calibration Plots (2020) https://twiki.cern.ch/twiki/bin/view/AtlasPublic/ApprovedPlotsTileEnergyCalibration.

[14] The ATLAS Collaboration, Public Tile Calorimeter Single Particle Response Plots (2020) https://twiki.cern.ch/twiki/bin/view/AtlasPublic/ApprovedPlotsTileSingleParticleResponse. 